\title{
Pressure-induced formation of rhodium zigzag chains in the honeycomb rhodate $\mathrm{Li}_{2} \mathrm{RhO}_{3}$
}

\author{
V. Hermann, ${ }^{1}$ S. Biswas, ${ }^{2}$ J. Ebad-Allah,,${ }^{1,3}$ F. Freund, ${ }^{4}$ A. Jesche, ${ }^{4}$ A. A. Tsirlin, ${ }^{4}$ M. Hanfland,${ }^{5}$ D. Khomskii, ${ }^{6}$ P. Gegenwart, ${ }^{4}$ \\ R. Valentí, ${ }^{2}$ and C. A. Kuntscher ${ }^{1, *}$ \\ ${ }^{1}$ Experimentalphysik II, Augsburg University, 86159 Augsburg, Germany \\ ${ }^{2}$ Institut für Theoretische Physik, Goethe-Universität Frankfurt, 60438 Frankfurt am Main, Germany \\ ${ }^{3}$ Department of Physics, Tanta University, 31527 Tanta, Egypt \\ ${ }^{4}$ Experimentalphysik VI, Center for Electronic Correlations and Magnetism, Augsburg University, 86159 Augsburg, Germany \\ ${ }^{5}$ European Synchrotron Radiation Facility, Boîte Postale 220, 38043 Grenoble, France \\ ${ }^{6}$ II. Physikalisches Institut, Universität zu Köln, Zülpicher Strasse, 77, 50937 Köln, Germany
}

(Received 7 May 2019; published 28 August 2019)

\begin{abstract}
We use powder x-ray diffraction to study the effect of pressure on the crystal structure of the honeycomb rhodate $\mathrm{Li}_{2} \mathrm{RhO}_{3}$. We observe low-pressure $\left(P<P_{c 1}=6.5 \mathrm{GPa}\right)$ and high-pressure $\left(P>P_{c 2}=14 \mathrm{GPa}\right)$ regions corresponding to the monoclinic $C 2 / \mathrm{m}$ symmetry, while a phase mixture is observed at intermediate pressures. At $P>P_{c 2}$, the honeycomb structure becomes distorted and features short Rh-Rh bonds forming zigzag chains stretched along the crystallographic $a$ direction. This is in contrast to dimerized patterns observed in triclinic high-pressure polymorphs of $\alpha-\mathrm{Li}_{2} \mathrm{IrO}_{3}$ and $\alpha-\mathrm{RuCl}_{3}$. Density-functional theory calculations at various pressure conditions reveal that the observed rhodium zigzag-chain pattern is not expected under hydrostatic pressure but can be reproduced by assuming anisotropic pressure conditions.
\end{abstract}

DOI: 10.1103/PhysRevB.100.064105

\section{INTRODUCTION}

In recent years, $4 d$ and $5 d$ transition-metal compounds were intensively studied due to their extremely rich physics. In comparison to $3 d$ compounds, where the electronic correlation $U$ dominates over the spin-orbit coupling constant $\lambda_{\text {SOC }}$ and Hund's coupling $J_{\mathrm{H}}$, spin-orbit coupling (SOC) becomes more and more important for $4 d$ and $5 d$ transition-metal compounds, whereas the strength of electronic correlations decreases. The actual physics of these compounds thereby depends on a delicate balance between $U, \lambda_{\mathrm{SOC}}$, and $J_{\mathrm{H}}$, as well as the crystal structure. The class of layered honeycombtype $4 d$ and $5 d$ transition-metal compounds, such as $A_{2} M_{3}$ $(A=\mathrm{Li}, \mathrm{Na}$ and $M=\mathrm{Ir}, \mathrm{Rh})$ and $\alpha-\mathrm{RuCl}_{3}$, is especially interesting in this regard, as this delicate balance of parameters was discussed in terms of Kitaev physics and a possible spin-liquid state [1-9]. However, in $\mathrm{Na}_{2} \mathrm{IrO}_{3}, \alpha-\mathrm{Li}_{2} \mathrm{IrO}_{3}$, and $\alpha-\mathrm{RuCl}_{3}$ the quantum spin-liquid ground state is not realized since these materials were found to order magnetically at low temperatures [4,10-12].

As for $\mathrm{Li}_{2} \mathrm{RhO}_{3}$, its magnetic ground state is still under debate. No long-range magnetic order could be found down to $\approx 0.5 \mathrm{~K}$, but instead, at small magnetic fields spin freezing was observed below 6-7 K $[8,9]$, although it is suspected that the majority of magnetic moments form a fluctuating liquidlike state [9]. Whether this partial spin freezing is due to proximity to the Kitaev quantum spin-liquid ground state or due to unavoidable defects (antisite disorder and/or stacking faults) is still unclear $[9,13]$. However, ab initio and effective-model calculations showed that $\mathrm{Li}_{2} \mathrm{RhO}_{3}$ bears electronic structure

\footnotetext{
*christine.kuntscher@physik.uni-augsburg.de
}

similar to the iridates [14] and hosts anisotropic Kitaev interaction terms of the same magnitude as in $5 d$ iridates [13]. According to electrical resistivity measurements, $\mathrm{Li}_{2} \mathrm{RhO}_{3}$ is insulating at ambient pressure $[8,14]$.

Another interesting aspect of $\mathrm{Li}_{2} \mathrm{RhO}_{3}$ is its behavior under pressure, where honeycomb iridates $[15,16]$ and $\alpha-\mathrm{RuCl}_{3}$ $[17,18]$ become dimerized and, consequently, nonmagnetic. Previously [15], we showed that the size of the central ion, the strength of the spin-orbit coupling, electronic correlations, and Hund's coupling all act against the dimerization. In comparison to $\alpha-\mathrm{Li}_{2} \mathrm{IrO}_{3}, \lambda_{\mathrm{SOC}}$ in $\mathrm{Li}_{2} \mathrm{RhO}_{3}$ is expected to be lower, while the electronic correlations should be enhanced in $\mathrm{Rh}^{4+}$ compared to $\mathrm{Ir}^{4+}$, as screening by oxygen orbitals is reduced. Therefore, one generally expects a higher transition pressure in $\mathrm{Li}_{2} \mathrm{RhO}_{3}$ and a larger pressure range for tuning the putative Kitaev magnetism of this compound. Here, we show that this is the case and also that the pressure-induced transformations are very different from the dimerization observed in honeycomb iridates.

\section{METHODS}

\section{A. Experimental details}

A powder sample of $\mathrm{Li}_{2} \mathrm{RhO}_{3}$ was prepared by a solidstate reaction of $\mathrm{Li}_{2} \mathrm{CO}_{3}$ and $\mathrm{Rh}$ in oxygen flow at $850^{\circ} \mathrm{C}$ with several intermediate regrindings. The sample quality was confirmed by laboratory $\mathrm{x}$-ray diffraction using the Rigaku MiniFlex diffractometer $(\mathrm{Cu} K \alpha$ radiation, Bragg-Brentano geometry). This synthesis procedure yield samples with the best structural order achieved so far [19], although stacking faults are still present. Their concentration is discussed in Sec. III A. 
$\mathrm{Li}_{2} \mathrm{RhO}_{3}$ powder was loaded into a diamond anvil cell (DAC) for pressure generation, and helium was used as a pressure-transmitting medium. The powder x-ray diffraction patterns were obtained using synchrotron radiation at beamline ID15B at the European Synchrotron Radiation Facility (ESRF), Grenoble, at room temperature. The wavelength of the radiation was $0.411267 \AA$, and the patterns were obtained in the $2 \theta$ range between $2^{\circ}$ and $33^{\circ}$. The pressure in the DAC was determined in situ by the ruby luminescence method. The resulting patterns were analyzed by Rietveld refinements using the JANA2006 software [20]. The quality of the fit is gauged by the profile factor $R_{p}$, as defined in Ref. [21], and by the commonly used weighted profile factor $R_{w p}=$ $\left\{\sum_{i} w_{i}\left[y_{i}^{\prime}(\mathrm{obs})-y_{i}^{\prime}(\mathrm{calc})\right]^{2}\right\} /\left[\sum_{i} w_{i} y_{i}^{\prime}(\mathrm{obs})^{2}\right]$, where $y_{i}^{\prime}$ are intensities corrected for background. The absorption correction for a cylindrical sample was calculated to be lower than the value 1 (using Ref. [22]), so no absorption correction was applied. The isotropic atomic displacement parameters $U_{\text {iso }}$ were fixed to the value of $0.005 \AA^{2}$ for all atomic positions, except for $\operatorname{Rh}(1)$ and $\operatorname{Li}(1)$.

\section{B. Computational details}

Structural optimizations were performed under different pressure conditions by using the projector augmented plane wave [23] method based on density-functional theory (DFT), as implemented in the VASP package [24]. Calculations were done within the generalized gradient approximation (GGA), $\mathrm{GGA}+U$ [25], and GGA $+\mathrm{SOC}+U$ (including spin-orbit coupling effects for $\mathrm{Rh}$ ). The value of the on-site Coulomb parameter $U$ was chosen based on the reproducibility of the experimental structure, as will be shown below. The cutoff for the wave function was set at $650 \mathrm{eV}$. $K$-point meshes of size $8 \times 6 \times 8$ were used for all the structural optimizations.

We performed two types of structural optimizations: (i) allowing relaxation of both lattice parameters and atomic positions under fixed hydrostatic pressure, which we refer to as "full relaxation," and (ii) keeping the lattice parameters fixed according to given pressure conditions and allowing the relaxation of only the atomic positions. In both cases, the system is allowed to relax until the total force acting on the system is less than $0.005 \mathrm{eV} / \AA$. At each pressure value, several different initial magnetic configurations were considered: (i) ferromagnetic (FM), (ii) zigzag antiferromagnetic (AFM), (iii) Neél AFM, (iv) stripy AFM, and (v) nonmagnetic (see Fig. 1).

The analysis of the electronic properties was done with the full-potential local orbital basis [26].

\section{RESULTS AND DISCUSSION}

\section{A. Experimental results}

The x-ray powder diffraction diagrams under pressure are displayed in Fig. 2. Additionally, we show the diffraction patterns at ambient pressure and at the highest studied pressure $(25.2 \mathrm{GPa})$ together with the corresponding fits from the Rietveld refinement in Fig. 3. Both refinements were performed within the monoclinic unit cell with $C 2 / m$ symmetry. The same crystal symmetry is found for the closely related honeycomb iridates $[4,27,28]$. In the refinements, stacking

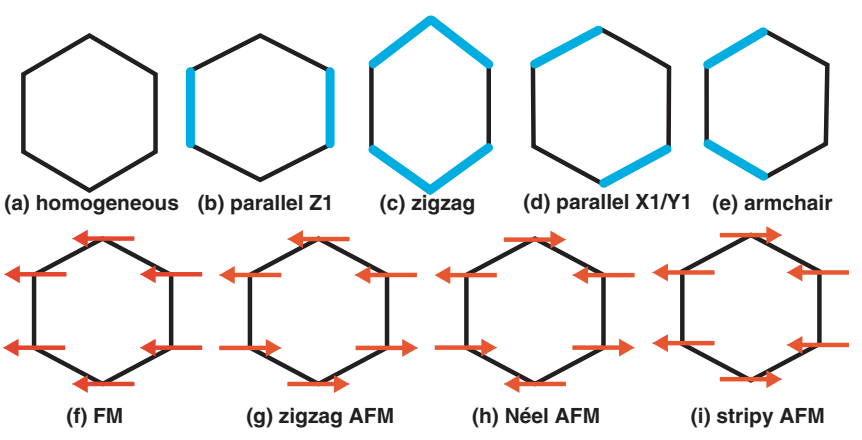

FIG. 1. Schematics of (a)-(e) various types of possible dimerization in hexagonal Kitaev systems and (f)-(i) different magnetic configurations considered by us for $\mathrm{Li}_{2} \mathrm{RhO}_{3}$. The blue lines indicate the short bond $l_{s}$, i.e., the dimer, and the red arrows indicate the spin orientation at the transition-metal site.

faults associated with shifts between successive $\mathrm{LiRh}_{2}$ layers were taken into account, as observed in $\alpha-\mathrm{Li}_{2} \mathrm{IrO}_{3}[27,28]$ and other $\mathrm{Li}_{2} M \mathrm{O}_{3}(M=\mathrm{Mn}, \mathrm{Pt}, \mathrm{Ru})$ compounds [27,29,30]. Stacking faults affect the intensity and line shape of several peaks and lead to an additional intensity between the (020) and (110) peaks, as marked by the dashed red arrows in the insets of Fig. 3. The presence of stacking faults was taken into account by introducing the $\mathrm{Li} / \mathrm{Rh}$ mixing for the $\mathrm{Rh}(1) / \mathrm{Li}(1)$ and $\mathrm{Li}(2) / \mathrm{Rh}(2)$ sites while constraining the overall stoichiometry to $\mathrm{Li}_{2} \mathrm{RhO}_{3}$. This reproduces the peak intensity but not its shape [27].

The lattice parameters as a function of pressure, as obtained by the Rietveld fits of the x-ray powder diffraction diagrams, are depicted in Fig. 4. Up to the critical pressure

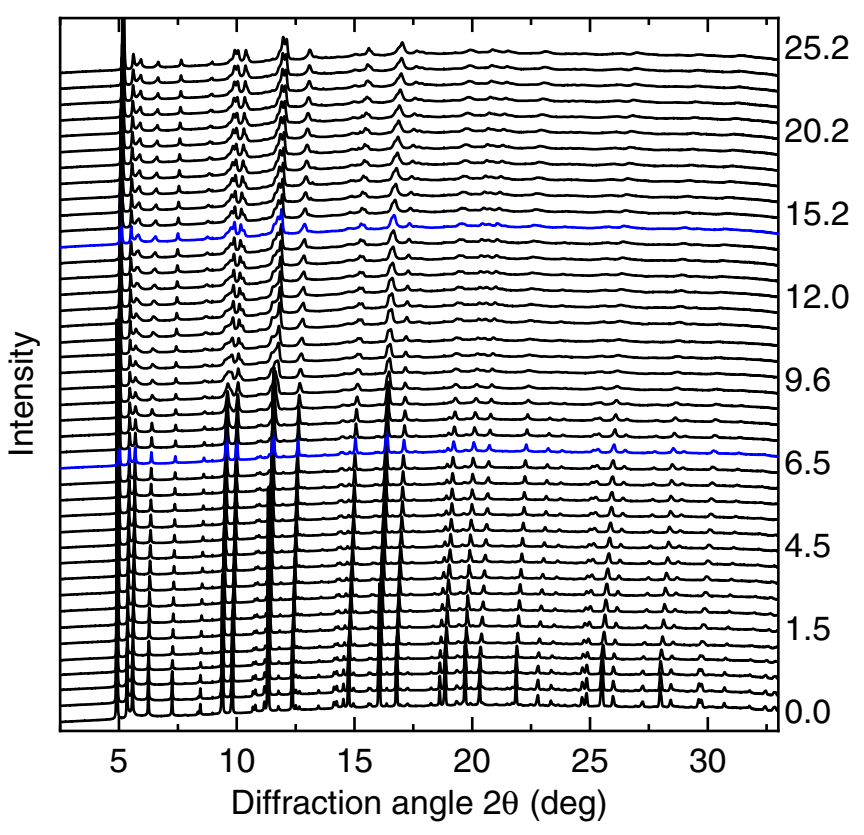

FIG. 2. Room-temperature $\mathrm{x}$-ray powder diffraction diagrams of $\mathrm{Li}_{2} \mathrm{RhO}_{3}$ under pressure. The numbers on the right vertical axis denote the applied pressures in gigapascals. The diffraction diagrams at the critical pressures $P_{c 1}=6.5 \mathrm{GPa}$ and $P_{c 2}=14 \mathrm{GPa}$ are highlighted by blue lines. 


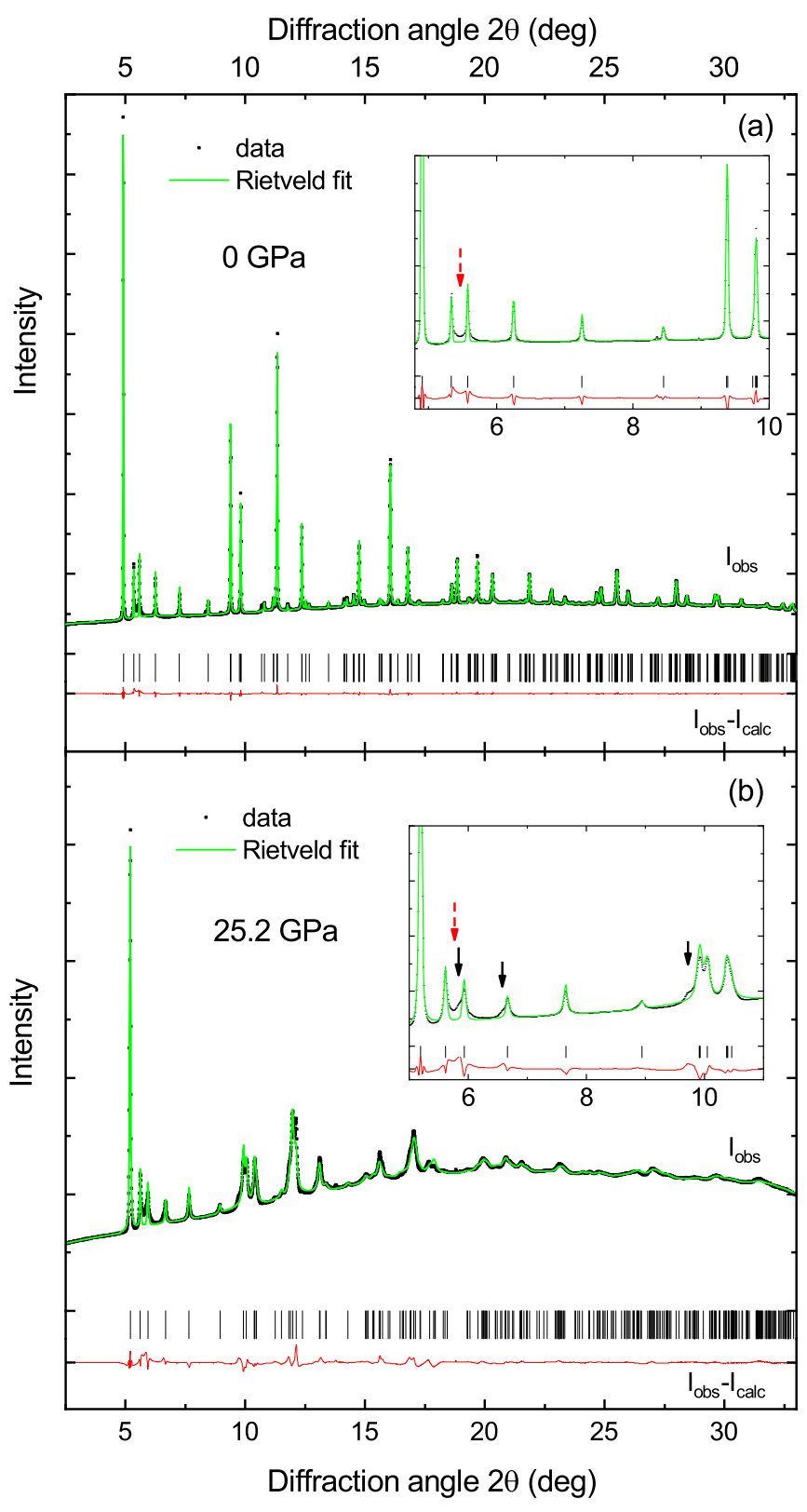

FIG. 3. X-ray powder diffraction diagrams $I_{\text {obs }}$ of $\mathrm{Li}_{2} \mathrm{RhO}_{3}$ at (a) the ambient pressure and (b) the highest studied pressure (25.2 GPa) together with the corresponding Rietveld fits $I_{\text {calc }}$ and the difference curves $\left(I_{\mathrm{obs}}-I_{\text {calc }}\right)$. Markers indicate the calculated peak positions. The $R_{p}\left(R_{w p}\right)$ values amount to $6.31 \%(13.30 \%)$ and $6.15 \%$ $(19.33 \%)$, respectively. The insets in (a) and (b) show the respective low-angle region at 0 and $25.2 \mathrm{GPa}$. The dashed red arrows in the insets mark the additional intensity due to stacking faults, while the black arrows in the inset of (b) mark traces from the low-pressure phase as discussed in the text.

$P_{c 1}=6.5 \mathrm{GPa}$, the lattice parameters $a, b^{\prime}=b / \sqrt{3}$, and $c$ decrease monotonically with increasing pressure in a very similar manner. The $c / a$ value, shown in the inset of Fig. 4(a), reveals that the strongest pressure-induced effect occurs for the lattice parameter $c$. The monoclinic angle $\beta$ decreases slightly but monotonically within this pressure range.

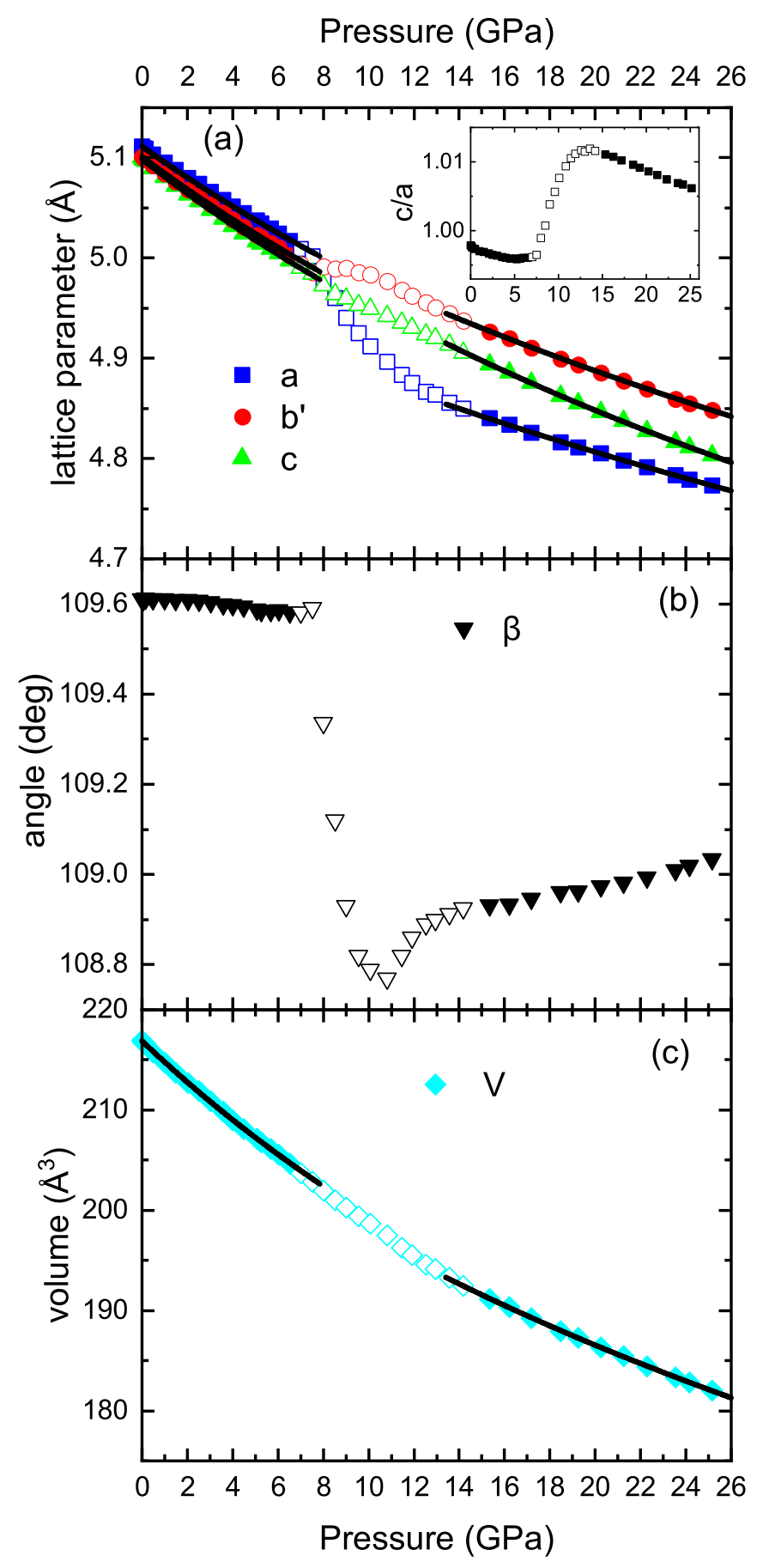

FIG. 4. Pressure evolution of (a) the lattice parameters $\left(a, b^{\prime}=\right.$ $b / \sqrt{3}, c$ ) and $c / a$ value (inset), (b) the monoclinic angle $\beta$ and $b / a$ value (inset, dashed line at $b / a=\sqrt{3}$ ), and (c) the volume $V$ of the unit cell. The solid lines are fits with a Murnaghan equation of state as explained in the text. Open symbols mark the intermediate pressure regime, where the results may be less accurate due to the phase mixture (see text).

Above $P_{c 1}$, a second phase with the same $C 2 / m$ symmetry appears and gets more pronounced with increasing pressure. The coexistence of two phases above $P_{c 1}$ suggests the firstorder character of the phase transition. Above the critical pressure $P_{c 2}=14 \mathrm{GPa}$, this second phase is dominant, and the 


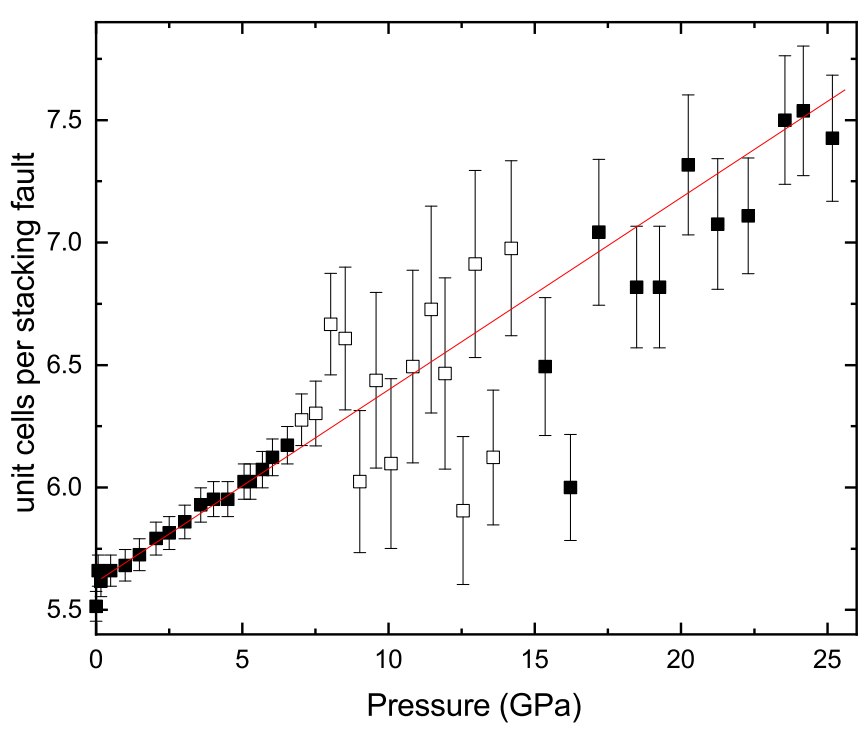

FIG. 5. Average number of unit cells per one stacking fault, as estimated from the fractional occupation of the $\mathrm{Li}(1) / \mathrm{Rh}(1)$ site. The red line is a guide to the eye.

high-pressure diffractograms can be well described by a single phase with $C 2 / m$ symmetry. There are only traces of the lowpressure phase found in the diffraction patterns above $P_{c 2}$ and up to the highest studied pressure, marked with black arrows in the inset of Fig. 3(b). Most importantly, we can rule out a symmetry lowering above $P_{c 2}$, as such a symmetry lowering would induce peak splittings, for example, for the (021) and the (111) diffraction peaks. These peaks are observed at $7.7^{\circ}$ and $9.0^{\circ}$ in the inset of Fig. 3(b) and are obviously not split. Thus, both the low-pressure $\left(P<P_{c 1}\right)$ and high-pressure $(P>$ $P_{c 2}$ ) phases in $\mathrm{Li}_{2} \mathrm{RhO}_{3}$ have $C 2 / m$ symmetry. This result is in contrast to the recent findings for $\alpha-\mathrm{Li}_{2} \mathrm{IrO}_{3}$, where a pressure-induced structural phase transition with symmetry lowering from monoclinic to triclinic symmetry caused by the Ir-Ir dimerization occurs at $3.8 \mathrm{GPa}$ [15]. Analogously, the monoclinic to triclinic symmetry lowering with the Ru-Ru dimerization is observed in $\alpha-\mathrm{RuCl}_{3}$ at $P \approx 1 \mathrm{GPa}$ [17].

The refinement of the diffraction patterns for the intermediate pressure range, $P_{c 1}<P<P_{c 2}$, with a phase mixture of the low-pressure and high-pressure phases did not yield stable fits, as many of the peaks of the two phases are broad and overlapping. Since the refinement with only one phase does not reproduce the actual peak shape, we marked this range with open symbols in Figs. 4, 5, 6, 7, and 8.

Between $P_{c 1}$ and $P_{c 2}$, lattice parameter $a$ decreases drastically by about $3 \%$, while there is only a slight but abrupt increase in lattice parameter $b$, and $c$ follows the pressureinduced monotonic decrease as observed below $P_{c 1}$ [see Fig. 4(a)]. The abrupt decrease in the $a$ parameter is also revealed by the abrupt increase in the $c / a$ ratio. Accordingly, the most pronounced pressure-induced change happens along the $a$ lattice direction, as will be discussed in more detail later. The monoclinic angle $\beta$ abruptly decreases above $P_{c 1}$, and above $P_{c 2}$ it monotonically increases with increasing pressure [see Fig. 4(b)]. The kink in the pressure evolution of $\beta$ in the pressure range $10-12 \mathrm{GPa}$, i.e., in the intermediate

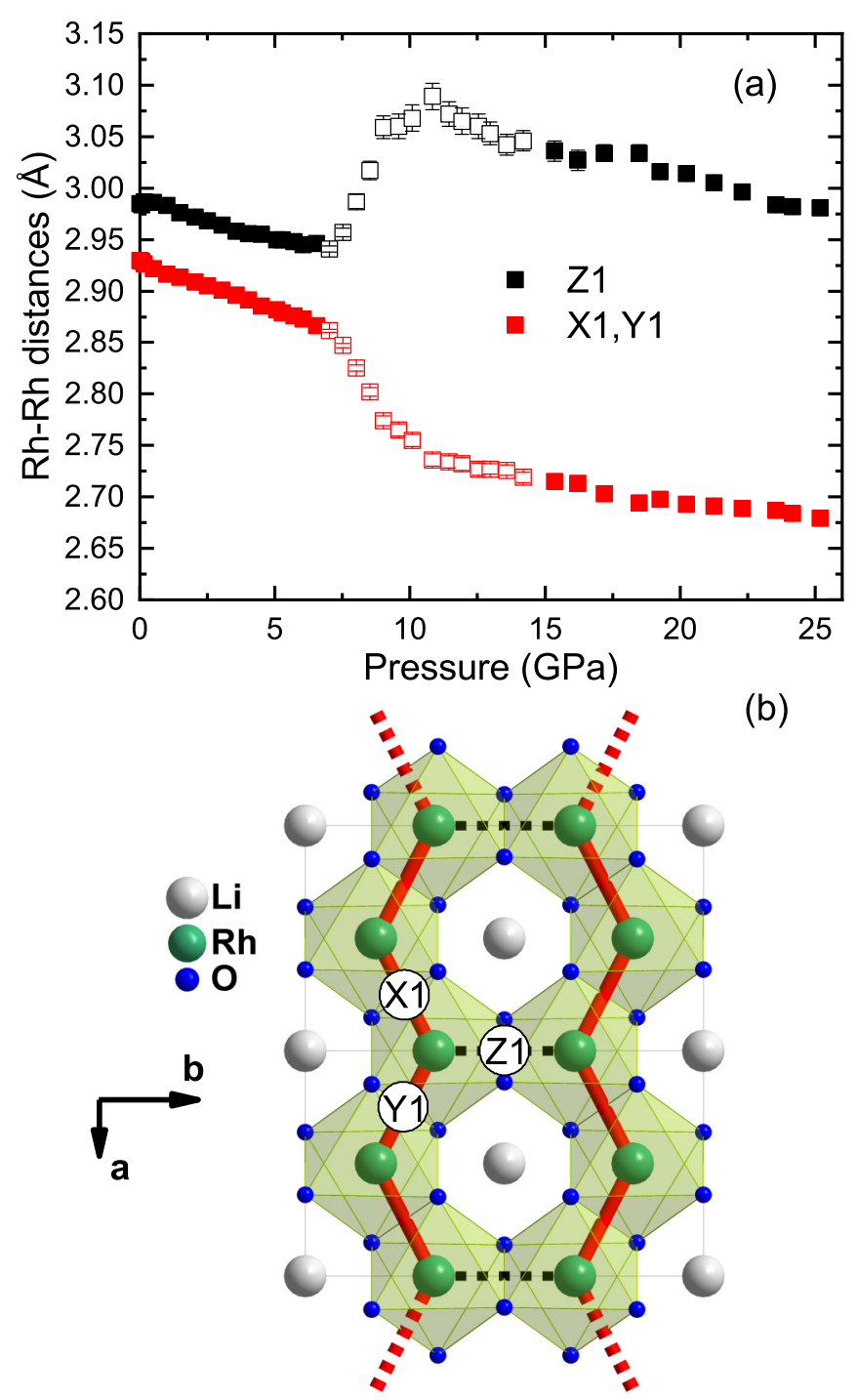

FIG. 6. (a) Rh-Rh bond lengths as a function of pressure for the rhodium hexagons in the $a b$ plane with the nomenclature (Rh bonds $X 1, Y 1, Z 1)$ given in (b). The ratio $l_{l} / l_{s}$ is calculated to $\approx 1.02$ and $\approx 1.11$ in the low- and high-pressure phases, respectively. The Rh zigzag chains along the $X 1$ and $Y 1$ bonds above $P_{c 2}$ are illustrated in (b) by thick red lines.

phase, is not discussed here because the phase mixture affects the refinements in this pressure range.

The pressure dependencies of the volume $V$ and the lattice parameters $r(r=a, b, c)$ were fitted separately for the lowand high-pressure phases, neglecting the intermediate regime, with a second-order Murnaghan equation of state (MOS) [31] to obtain the bulk moduli $B_{0, V}$ and $B_{0, r}$ according to

$$
\begin{gathered}
V(P)=V_{0}\left[\left(B_{0}^{\prime} / B_{0, V}\right) P+1\right]^{-1 / B_{0}^{\prime}}, \\
r(P)=r_{0}\left[\left(B_{0}^{\prime} / B_{0, r}\right) P+1\right]^{-1 / 3 B_{0}^{\prime}},
\end{gathered}
$$

with $B^{\prime}$ fixed to 4 . The results are summarized in Table I. The bulk modulus $B_{0, V}$ of the low- and high-pressure phases amounts to 100.4(4) and 118.6(9) GPa, respectively. This means that the material is less compressible in the highpressure phase. In the low-pressure phase $\left(P<P_{c 1}\right)$, the 

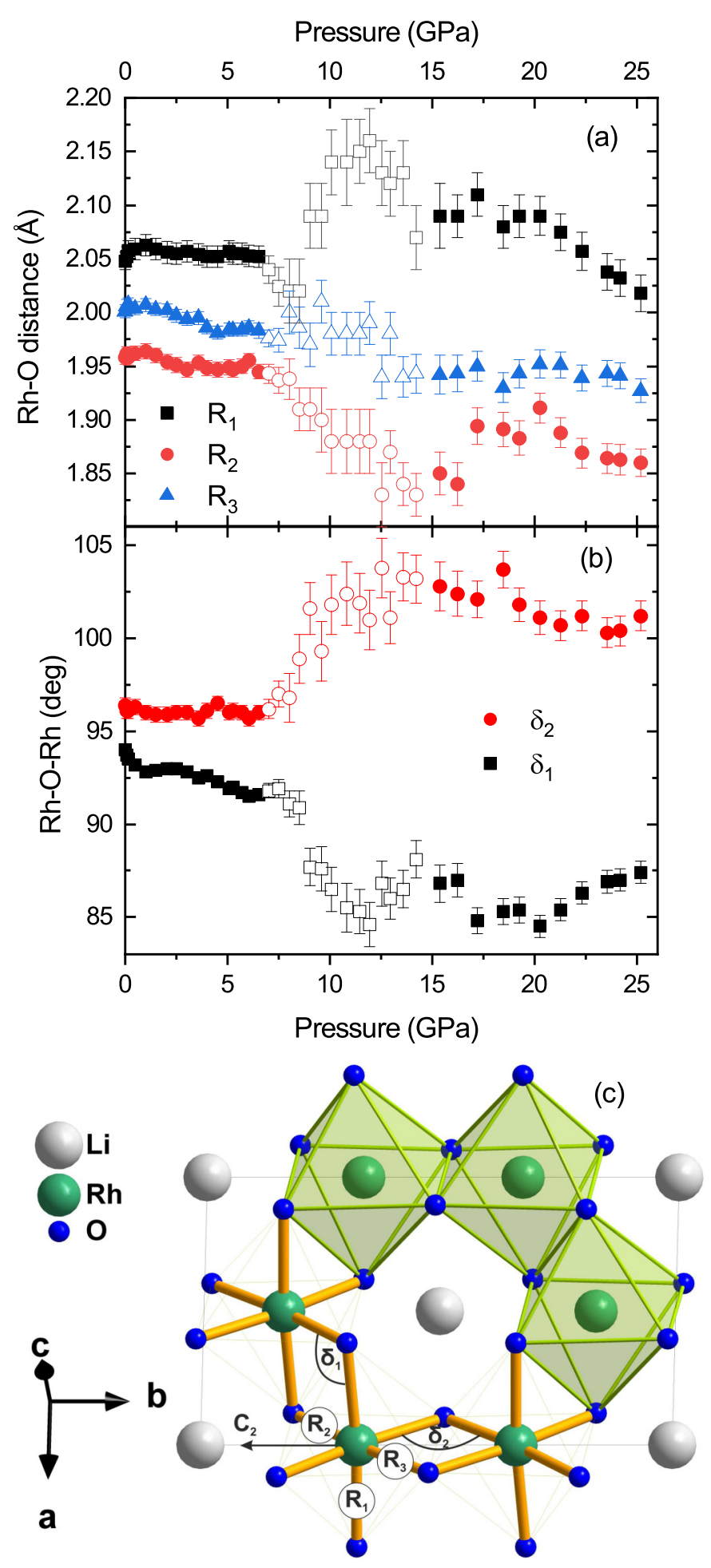

FIG. 7. Pressure dependence of the various octahedral (a) Rh-O distances $\left(R_{1}, R_{2}, R_{3}\right)$ and (b) Rh-O-Rh bond angles $\left(\delta_{1}, \delta_{2}\right)$ with the nomenclature given in (c). The $C_{2}$ rotational axis is indicated by an arrow.

contribution of the $c$ direction to the bulk modulus is the lowest, with $B_{0, c}=94.8(7) \mathrm{GPa}$, as already indicated by the pressure dependence of the $c / a$ ratio [inset of Fig. 4(a)]. Thus, the material is most compressible along the $c$ direction. The

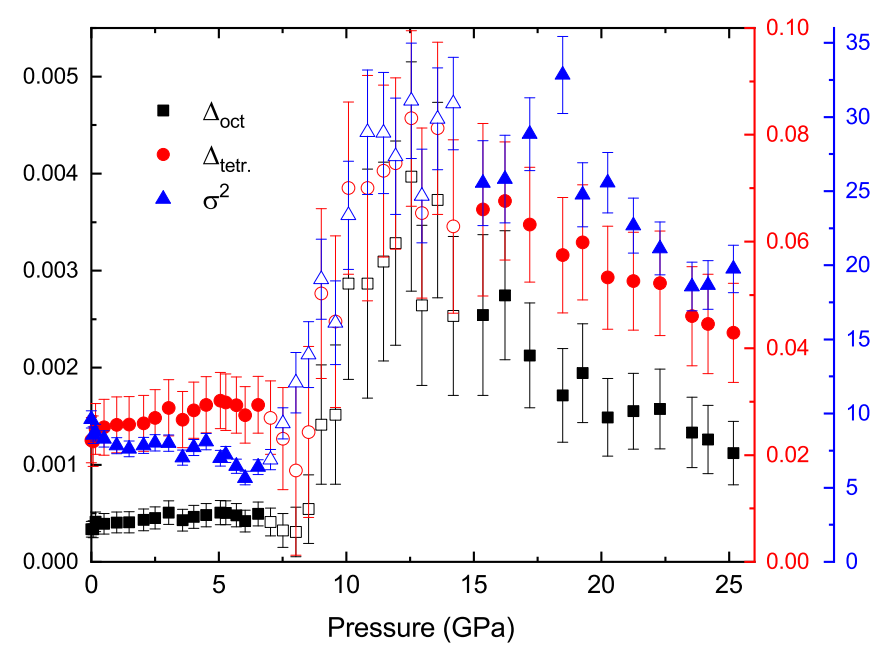

FIG. 8. Pressure dependence of the bond-length distortion $\Delta_{\text {oct }}$, the tetragonal distortion $\Delta_{\text {tetr }}$, and the bond-angle distortion $\sigma_{\text {oct }}^{2}$ as defined in the text.

largest contribution to the bulk modulus is attributed to the $a$ crystal direction, with $B_{0, a}=105.7(5) \mathrm{GPa}$.

In the high-pressure phase $\left(P>P_{c 2}\right)$, the contribution $B_{0, c}$ of the $c$ direction remains low and is even slightly decreased compared to the low-pressure phase. Most interestingly, the contribution $B_{0, a}$ is strongly increased to $155.6(15) \mathrm{GPa}$ in the high-pressure phase, while $B_{0, b}$ is much less increased, i.e., to 122.2(12) GPa. Hence, the honeycomb layers along the $a b$ plane become less compressible in the high-pressure phase, whereby the pressure-induced hardening has the strongest effect along the $a$ direction.

For a more detailed discussion, the atomic parameters of the refinement are shown in Table II. The partial exchange of $\mathrm{Li}$ and $\mathrm{Rh}$ accounts for the stacking faults, as described in Ref. [27]. Since three Rh atoms are required to change place with one $\mathrm{Li}$ atom in order to mimic one stacking fault and taking into account the site symmetry of the $\mathrm{Rh}(1)$ position $(4 g)$, the number of unit cells per stacking fault amounts to $3 /\left(4 \mathrm{occ}_{\mathrm{Li}(1)}\right)$, where occ $\mathrm{Li}_{\mathrm{Li}(1)}$ denotes the $\mathrm{Li}(1)$ site occupancy. Accordingly, every 5.5(1) unit cells (along the $c$ direction) one stacking fault occurs at ambient pressure. This value is very similar to previous reports on $\mathrm{Li}_{2} \mathrm{RhO}_{3}[8,32]$ and slightly higher than in $\alpha-\mathrm{Li}_{2} \mathrm{IrO}_{3}$ and $\mathrm{Li}_{2} \mathrm{PtO}_{3}$ [27]. On the other hand, different studies of $\mathrm{Na}_{2} \mathrm{IrO}_{3}[4,33]$ reported concentrations of stacking faults that are either larger or smaller than in

TABLE I. Bulk moduli $B_{0, V}$ and $B_{0, r}$, with $r=a, b, c$, in the lowpressure $(P<6.5 \mathrm{GPa})$ and high-pressure $(P>14 \mathrm{GPa})$ phases, as obtained from fitting the volume $V$ and lattice parameters $r$ with a MOS, with $B_{0}^{\prime}$ set to 4 .

\begin{tabular}{lcc}
\hline \hline & $P<6.5 \mathrm{GPa}$ & $P>15 \mathrm{GPa}$ \\
\hline$V_{0}\left(\AA^{3}\right)$ & $216.90(3)$ & $212.21(16)$ \\
$B_{0, V}(\mathrm{GPa})$ & $100.4(4)$ & $118.6(9)$ \\
$B_{0, a}(\mathrm{GPa})$ & $105.7(5)$ & $155.6(15)$ \\
$B_{0, b}(\mathrm{GPa})$ & $100.2(6)$ & $122.2(12)$ \\
$B_{0, c}(\mathrm{GPa})$ & $94.8(7)$ & $93.9(8)$ \\
\hline \hline
\end{tabular}


TABLE II. Structural parameters for the low-pressure phase at ambient pressure and for the high-pressure phase at $25.2 \mathrm{GPa}$. At ambient pressure, the lattice parameters are $a=5.11126(10) \AA, b=8.83473(16) \AA, c=5.10034(11) \AA, \beta=109.6105(18)^{\circ}$, and $V=216.955(8) \AA^{3}$, and at $25.2 \mathrm{GPa}$ they are $a=4.7732(5) \AA, b=8.3980(7) \AA, c=4.8027(3) \AA, \beta=109.034(11)^{\circ}$, and $V=181.99(3) \AA^{3}$. The isotropic atomic displacement parameters $U_{\text {iso }}$ were fixed to $0.005 \AA^{2}$ for all atomic positions, except for the $\operatorname{Rh}(1) / \operatorname{Li}(1)$ one.

\begin{tabular}{|c|c|c|c|c|c|c|c|c|c|c|c|}
\hline \multirow[b]{2}{*}{ Atom } & \multirow[b]{2}{*}{ Site } & \multicolumn{5}{|c|}{ Low-pressure phase (0 GPa) } & \multicolumn{5}{|c|}{ High-pressure phase (25.2 GPa) } \\
\hline & & $x$ & $y$ & $z$ & Occupancy & $U_{\text {iso }}\left(\AA^{2}\right)$ & $x$ & $y$ & $z$ & Occupancy & $U_{\text {iso }}\left(\AA^{2}\right)$ \\
\hline $\operatorname{Rh}(1)$ & $4 g$ & 0 & $0.3311(2)$ & 0 & $0.864(3)$ & $0.0029(3)$ & 0 & $0.3225(5)$ & 0 & $0.899(7)$ & $0.0047(11)$ \\
\hline $\operatorname{Li}(1)$ & $4 g$ & 0 & $0.3311(2)$ & 0 & $0.136(3)$ & $0.0029(3)$ & 0 & $0.3225(5)$ & 0 & $0.101(7)$ & $0.0047(11)$ \\
\hline $\operatorname{Li}(2)$ & $2 a$ & 0 & 0 & 0 & $0.728(3)$ & 0.005 & 0 & 0 & 0 & $0.798(7)$ & 0.005 \\
\hline $\operatorname{Rh}(2)$ & $2 a$ & 0 & 0 & 0 & $0.273(3)$ & 0.005 & 0 & 0 & 0 & $0.202(7)$ & 0.005 \\
\hline $\operatorname{Li}(3)$ & $4 h$ & 0 & $0.820(3)$ & 0.5 & 1 & 0.005 & 0 & $0.808(8)$ & 0.5 & 1 & 0.005 \\
\hline $\operatorname{Li}(4)$ & $2 d$ & 0 & 0.5 & 0.5 & 1 & 0.005 & 0 & 0.5 & 0.5 & 1 & 0.005 \\
\hline $\mathrm{O}(1)$ & $8 j$ & $0.252(17)$ & $0.3209(7)$ & $0.7631(10)$ & 1 & 0.005 & $0.271(3)$ & $0.3332(16)$ & $0.754(3)$ & 1 & 0.005 \\
\hline $\mathrm{O}(2)$ & $4 i$ & $0.274(2)$ & 0 & $0.7726(19)$ & 1 & 0.005 & $0.287(4)$ & 0 & $0.774(4)$ & 1 & 0.005 \\
\hline
\end{tabular}

$\mathrm{Li}_{2} \mathrm{RhO}_{3}$. The number of unit cells per stacking fault increases monotonically with increasing pressure and reaches 7.4(5) at 25.2 GPa, as shown in Fig. 5; that is, the number of stacking faults is slightly reduced by external pressure. The parameters for the oxygen positions are changed in the high-pressure phase compared to the low-pressure phase, thus affecting the $\mathrm{RhO}_{6}$ octahedra. The most interesting change, however, is observed for the $y$ parameter of $\operatorname{Rh}(1)$ that determines the $\mathrm{Rh}-\mathrm{Rh}$ distances in the honeycomb network (see Table II).

To evaluate this behavior further, we compare the pressure evolution of the three Rh-Rh bond lengths in the $a b$ plane, namely, the $Z 1$ bond and the two degenerate $X 1 / Y 1$ bonds, as depicted in Fig. 6(b). At ambient pressure, the $Z 1$ bond length amounts to $2.985(3) \AA$, while the $X 1 / Y 1$ bond length is 2.9296(13) $\AA$ [see Fig. 6(a)], leading to a slightly distorted honeycomb. The corresponding bond disproportionation $l_{l} / l_{s}$, with $l_{l}$ and $l_{s}$ being the long and short bonds of the hexagonal $\mathrm{Rh}$ network, respectively, amounts to $l_{l} / l_{s}=1.02$. For the high-pressure phase, both $X 1$ and $Y 1$ bonds are drastically reduced by $\approx 0.15 \AA$, while the $Z 1$ bond is increased by the same amount [Fig. 6(a)]. Hence, the bond disproportionation increased to $l_{l} / l_{s}=1.11$ at $25 \mathrm{GPa}$. The $X 1 / Y 1$ bond length of $\approx 2.7 \AA$ above $P_{c 2}$ is close to but still larger than the interatomic distances in metallic rhodium $(d=2.69 \AA[34])$. We thus conclude that external pressure introduces zigzag chains of rhodium atoms along the $a$ direction, as illustrated in Fig. 6(b). A similar structure but with closer bond lengths is found for $5 \%$ Na-doped crystals $\left(\mathrm{Li}_{0.95} \mathrm{Na}_{0.05}\right)_{2} \mathrm{RuO}_{3}$ at ambient pressure [35]. On the other hand, pure $\mathrm{Li}_{2} \mathrm{RuO}_{3}$ is dimerized at ambient pressure with an armchair pattern of the short Ru-Ru bonds [see Fig. 1(e) for illustration] [35,36].

Next, we consider the pressure-induced changes in the $\mathrm{RhO}_{6}$ octahedra. To this end, we define various $\mathrm{Rh}-\mathrm{O}$ bond lengths and $\mathrm{Rh}-\mathrm{O}-\mathrm{Rh}$ bond angles that are responsible for the direct metal-to-metal and indirect oxygen-mediated contributions. The octahedra possess a twofold rotational $C_{2}$ axis which is indicated by the arrow in Fig. 7(c). There are three unique $\mathrm{Rh}-\mathrm{O}$ bonds labeled $R_{1}, R_{2}, R_{3}$ and two unique $\mathrm{Rh}-\mathrm{O}-$ $\mathrm{Rh}$ angles $\delta_{1}$ and $\delta_{2}$, where $\delta_{1}\left(\delta_{2}\right)$ involves two Rh atoms connected via the $X 1 / Y 1$ bond ( $Z 1$ bond) [see Fig. 7(c)]. The pressure dependence of the various bonds and bond angles is depicted in Figs. 7(a) and 7(b), respectively. At ambient pressure, the largest Rh-O bond length is found for the apical oxygen atom; thus, the $\mathrm{RhO}_{6}$ octahedra show a tetragonal distortion with axial elongation. In the low-pressure phase $\left(P<P_{c 1}\right)$, the bond length $R_{1}$ is pressure independent, whereas $R_{2}$ and $R_{3}$ slightly decrease under pressure.

At $P_{c 2}$, the length $R_{1}$ is increased compared to the lowpressure phase, whereas $R_{2}$ and $R_{3}$ are decreased. Upon further compression, $R_{1}$ decreases, $R_{3}$ seems to be unaffected, and $R_{2}$ shows a small anomaly at 15-20 GPa that may be significant, as the changes exceed the error bars.

The formation of zigzag chains is predominantly due to a change in $\mathrm{Rh}-\mathrm{O}-\mathrm{Rh}$ angles as described in the following. The pressure dependence of the Rh-O-Rh bond angles $\delta_{1}$ and $\delta_{2}$ is shown in Fig. 7(b). At ambient pressure, the values of $\delta_{1}$ and $\delta_{2}$ amount to $94.0(3)^{\circ}$ and $96.4(4)^{\circ}$, respectively. While $\delta_{2}$ is independent of pressure in the low-pressure phase, $\delta_{1}$ decreases by increasing pressure. When entering the high-pressure phase above $P_{c 2}$ the bond angle $\delta_{1}$ is strongly decreased to the value $87^{\circ}$. Interestingly, the onset of the intermediate phase at $P_{c 1}$ appears at a pressure when $\delta_{1}$ approaches $90^{\circ}$, which is a distinct angle for the contributions of the ligand-mediated hopping to the hopping parameters, as discussed in more detail in Refs. [7,37]. The strong pressureinduced decrease in the angle $\delta_{1}$ between $P_{c 1}$ and $P_{c 2}$ confirms the formation of Rh zigzag chains along the $a$ direction. Consistently, the bond angle $\delta_{2}$ is strongly increased, as the $Z_{1}$ bond length is increased [see Fig. 6(a)]. Again, an anomaly is observed for the Rh-O-Rh bond angles between 15 and $20 \mathrm{GPa}$, which is directly related to the anomaly for the Rh-O distances and thereby has the same origin.

The electronic states of $\mathrm{Li}_{2} \mathrm{RhO}_{3}$ are affected by the distortion of the $\mathrm{RhO}_{6}$ octahedra. Therefore, we followed the pressure dependence of the octahedral distortion using the bond-length distortion $\Delta_{\text {oct }}$ and the bond-angle distortion $\sigma_{\text {oct }}^{2}$ [38-40]. The bond-length distortion is defined as $\Delta_{\text {oct }}=$ $\frac{1}{6} \sum_{i=1}^{6}\left[\left(d_{i}-d_{\mathrm{av}}\right) / d_{\mathrm{av}}\right]^{2}$, where $d_{i}$ is an individual Rh-O bond length and $d_{\mathrm{av}}$ is the average $\mathrm{Rh}-\mathrm{O}$ bond length in the $\mathrm{RhO}_{6}$ octahedron. The bond-angle distortion is calculated according to $\sigma_{\text {oct }}^{2}=\frac{1}{11} \sum_{i=1}^{12}\left(\alpha_{i}-90\right)^{2}$, where $\alpha_{i}$ is an individual $\mathrm{O}$ $\mathrm{Rh}-\mathrm{O}$ bond angle. At ambient pressure, the distortion parameters are $\Delta_{\text {oct }}=3.4(11) \times 10^{-4}$ and $\sigma^{2}=9.6(6)$, comparable to the results in Ref. [32] $\left[\Delta_{\text {oct }}=1.2(6) \times 10^{-4}\right.$ and 
$\left.\sigma^{2}=9.6(5)\right]$, although the $\mathrm{Rh}$ position seems to be fixed in that report. Comparison of our refinement to previous ones $[8,14]$ is not straightforward since in those studies some oxygen parameters were fixed or calculated. The distortion parameters for $\mathrm{Li}_{2} \mathrm{RhO}_{3}$ reported in our study are comparable to the ones of the related materials $\alpha-\mathrm{Li}_{2} \mathrm{IrO}_{3}$ and $\mathrm{Li}_{2} \mathrm{PtO}_{3}$ $[27,28]$. For $\mathrm{Na}_{2} \mathrm{IrO}_{3}$ the $\Delta_{\text {oct }}$ value is about one magnitude smaller, while the bond-angle distortion $\sigma^{2}$ is nearly doubled $[4,33]$. A comparison to the octahedral distortions in dimerized $\mathrm{Li}_{2} \mathrm{RuO}_{3}$ is difficult since the reported values determined by various studies are not consistent. For example, the $\Delta_{\text {oct }}$ values between $1.4 \times 10^{-4}$ and $24 \times 10^{-4}$ have been reported, and the values for $\sigma^{2}$ range between 4.7 and 54 $[35,41,42]$.

In the low-pressure phase, the bond-length distortion only slightly increases with increasing pressure, whereas the bondangle distortion decreases (Fig. 8). At the critical pressure $P_{c 2}$, both parameters $\Delta_{\text {oct }}$ and $\sigma_{\text {oct }}^{2}$ are drastically enhanced compared to the low-pressure range. Such an enhanced distortion was also reported in Ref. [41] for dimerized $\mathrm{Li}_{2} \mathrm{RuO}_{3}$ compared to the nondimerized samples. It is therefore likely that the enhancement of $\Delta_{\text {oct }}$ and $\sigma_{\text {oct }}^{2}$ at $P_{c 2}$ is caused by the lattice strain due to the formation of the $\mathrm{Rh}-\mathrm{Rh}$ zigzag chains in $\mathrm{Li}_{2} \mathrm{RhO}_{3}$.

Of further interest is the tetragonal distortion (elongation or compression along the $z$ direction) of the octahedra, as this would cause a splitting of the $\mathrm{Rh} t_{2 g}$ states. As a measure of the tetragonal distortion we define the parameter $\Delta_{\text {tetr }}$ as the deviation of the apical $\mathrm{Rh}-\mathrm{O}$ bond length $R_{1}$ from the average $\mathrm{Rh}$ O bond length $d_{\mathrm{av}}$ according to $\Delta_{\text {tetr }}=\left(R_{1}-d_{\mathrm{av}}\right) / d_{\mathrm{av}}$ [39]. For positive (negative) nonzero values of $\Delta_{\text {tetr }}$ the octahedra are elongated (compressed) along the apical bond direction. Such a distortion can be explained by a cooperative first-order Jahn-Teller effect neglecting stress on the system $[43,44]$. The Jahn-Teller effect is expected to be weak but nonzero in a $d^{5}$ configuration. The pressure dependence of $\Delta_{\text {tetr }}$ is depicted in Fig. 8. We note that the tetragonal distortion at ambient pressure amounts to $\Delta_{\text {tetr }}=0.023(4)$, which is comparable to the value $\Delta_{\text {tetr }}=0.015$ (3) given in Ref. [32]. In the low-pressure phase, $\Delta_{\text {tetr }}$ increases slightly but steadily upon compression; that is, the elongation increases.

Between the low- and high-pressure phases, $\Delta_{\text {tetr }}$ is nearly doubled, before it decreases upon further compression above $P_{c 2}$. While the tetragonal distortion in the low-pressure phase is comparable to that in $\mathrm{Li}_{2} M \mathrm{O}_{3}(M=\mathrm{Ir}, \mathrm{Pt})$, it is much more pronounced than in $\mathrm{Na}_{2} \mathrm{IrO}_{3}$, where $\Delta_{\text {tetr }}$ is close to zero. We thus conclude that the lattice strain rather than the Jahn-Teller effect is the driving force for the distortion of the octahedra in the honeycomb lattices. The tetragonal distortion in the high-pressure phase of $\mathrm{Li}_{2} \mathrm{RhO}_{3}$ is comparable to the tetragonal distortion in the perovskites $\mathrm{Sr}_{2} \mathrm{RhO}_{4}$ and $\mathrm{Sr}_{2} \mathrm{RuO}_{4}$, where the bond angle distortion is zero $[45,46]$.

\section{B. Computational results}

A question that remains open is why $\mathrm{Li}_{2} \mathrm{RhO}_{3}$ retains the monoclinic $C 2 / m$ symmetry and shows the zigzag-chain pattern of short $\mathrm{Rh}-\mathrm{Rh}$ bonds under pressure, whereas $\alpha-\mathrm{Li}_{2} \mathrm{IrO}_{3}$ $[15,16]$ and $\alpha-\mathrm{RuCl}_{3}$ [17] become triclinic following the formation of metal-metal dimers.
In previous studies, the experimentally observed dimerization pattern in $\alpha-\mathrm{Li}_{2} \mathrm{IrO}_{3}$ and $\alpha-\mathrm{RuCl}_{3}$ was identified by DFT calculations within the GGA $+\mathrm{SOC}+U$ scheme [15] as a consequence of a complex interplay of SOC, magnetism, correlation, and covalent bonding. Following this knowledge, we performed full relaxations of $\mathrm{Li}_{2} \mathrm{RhO}_{3}$ as a function of hydrostatic pressure with and without SOC. As an initial guess for the geometrical optimization at each pressure, we considered two structures: the experimental low-pressure "undistorted" structure at $5 \mathrm{GPa}$ and the experimental high-pressure "zigzag chain" structure at $25.2 \mathrm{GPa}$. In our notation, we assume a structure is undistorted when the corresponding bond disproportionation $l_{l} / l_{s}<1.04$. Moreover, for each of these initial geometries, we considered five different spin configurations, as explained in Sec. II B.

Test calculations performed at $25 \mathrm{GPa}$ reveal that after relaxation the structure becomes dimerized, regardless of the initial configuration. At a given pressure, the energetics of the various different configurations are obtained by comparing the corresponding enthalpies. Due to the underbinding problem of GGA (relaxed interatomic distances are longer than their experimental counterparts), the volume corresponding to $2 \mathrm{GPa}$ reproduces the experimental volume at ambient pressure. This has been corrected by systematically subtracting $\Delta P=2 \mathrm{GPa}$ from all simulated pressure values.

The value of Hubbard correlation $U=1.5 \mathrm{eV}$ was chosen such that at $5 \mathrm{GPa}$ (within the $\mathrm{GGA}+\mathrm{SOC}+U$ scheme) (i) the optimized lowest-enthalpy magnetic configuration corresponds to $C 2 / m$ symmetry and reproduces the experimental value of $l_{l} / l_{s}=1.02$ and (ii) the nonmagnetic configuration is dimerized (though the parallel $X 1 / Y 1$ type). The latter confirms dimerization at finite pressure for $\mathrm{Li}_{2} \mathrm{RhO}_{3}$ as magnetism is known to work against dimerization by pushing the transition pressure to a higher value [15].

At a pressure $P_{c}^{\text {noSOC }} \approx 11 \mathrm{GPa}$, we find that $\mathrm{Li}_{2} \mathrm{RhO}_{3}$ undergoes a phase transition from a homogeneous to a dimerized phase with bond disproportionation $l_{l} / l_{s}=1.146$ within the GGA $+U$ scheme (not shown here). Upon dimerization, $\mathrm{Li}_{2} \mathrm{RhO}_{3}$ becomes nonmagnetic. However, there are a few discrepancies with the experimental structures: (i) below $P_{c}^{\text {noSOC }}$, in the homogeneous structure, the shorter bond corresponds to the $Z 1$ bond, rather than to the $X 1$ and $Y 1$ bonds as observed in experiments, and (ii) the dimerized phase does not have $C 2 / m$ symmetry; rather, it has triclinic $(P \overline{1})$ symmetry, similar to $\alpha-\mathrm{RuCl}_{3}[17]$ and $\alpha-\mathrm{Li}_{2} \mathrm{IrO}_{3}[15,16]$. The inclusion of SOC causes the shorter bond to be $X 1 / Y 1$ in the homogeneous phase (below $P_{c}^{\mathrm{SOC}}$ ) and shifts the transition pressure $P_{c m}$ to $27 \mathrm{GPa}$. However, the high-pressure structure still becomes triclinic with $l_{l} / l_{s}=1.15$ (see Fig. 9).

The above results show that the experimental zigzag pattern of the short Rh-Rh bonds cannot be obtained from hydrostatic pressure simulations. We therefore proceed by simulating uniaxial pressure with the $b$ and $c$ parameters fixed to their experimental values at $25.5 \mathrm{GPa}$ and the $b / a$ ratio varied systematically (Fig. 10). This approach yields the zigzag-chain structure observed experimentally. However, we had to increase the $b / a$ ratio to 1.95 in order to reproduce the ratio $l_{l} / l_{s}$ between the long and short bonds.

To obtain the pressure evolution of $\mathrm{Rh}-\mathrm{Rh}$ bonds under uniaxial condition, we next repeated the above calculations but 

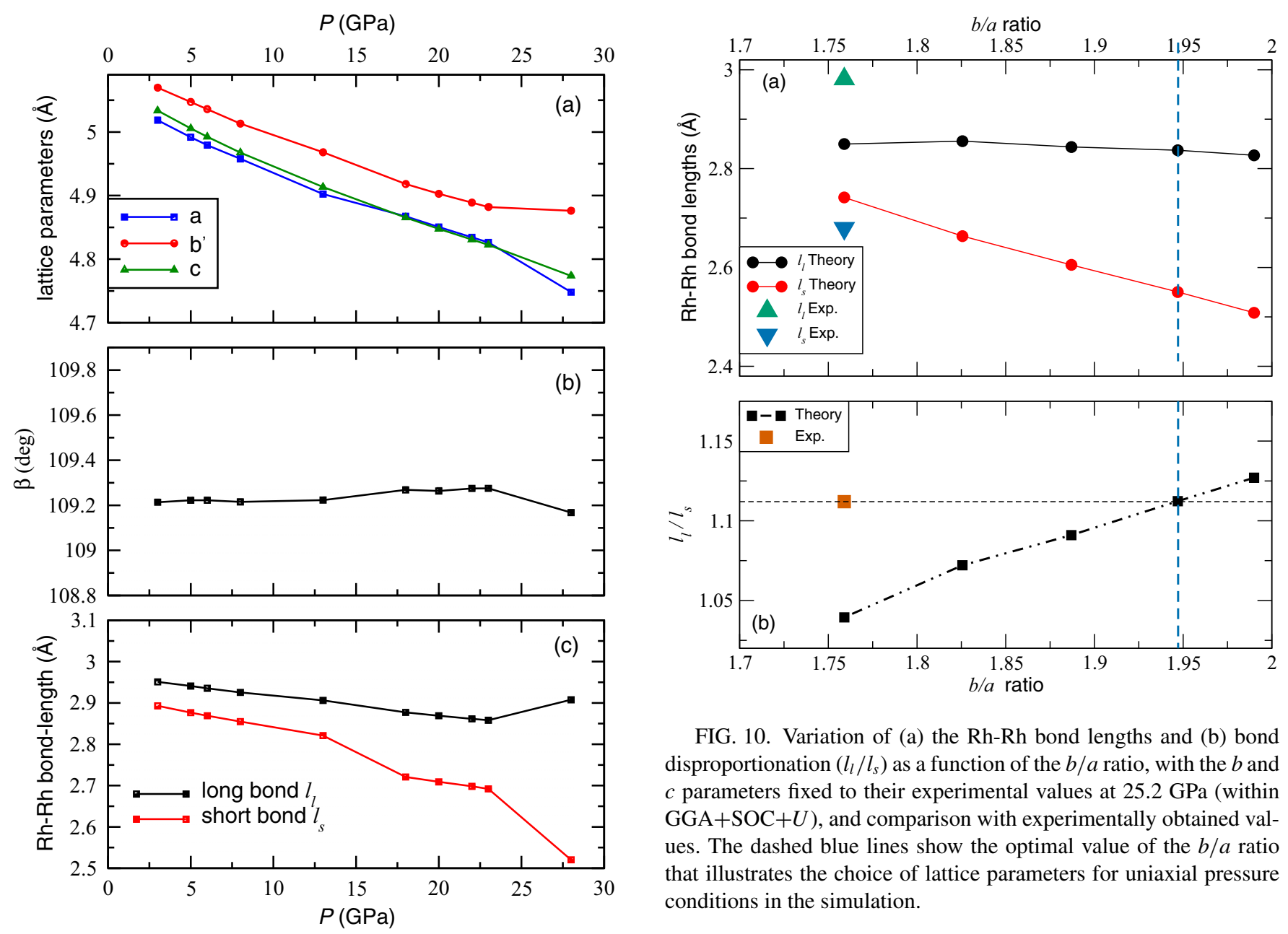

FIG. 10. Variation of (a) the Rh-Rh bond lengths and (b) bond disproportionation $\left(l_{l} / l_{s}\right)$ as a function of the $b / a$ ratio, with the $b$ and $c$ parameters fixed to their experimental values at $25.2 \mathrm{GPa}$ (within $\mathrm{GGA}+\mathrm{SOC}+U$ ), and comparison with experimentally obtained values. The dashed blue lines show the optimal value of the $b / a$ ratio that illustrates the choice of lattice parameters for uniaxial pressure conditions in the simulation.

The origin of the uniaxial-like pressure conditions requires

FIG. 9. Pressure dependence of the theoretically obtained (a) and (b) structural parameters calculated under hydrostatic pressure conditions and (c) Rh-Rh bond lengths within the GGA $+\mathrm{SOC}+U(U=$ $1.5 \mathrm{eV})$ scheme.

this time with $b / a=1.95$ while fixing the $b$ and $c$ parameters to their experimental values at the corresponding pressure. By comparing the results of the hydrostatic and uniaxial pressure simulations, we conclude (see Fig. 11) that the evolution of $\mathrm{Li}_{2} \mathrm{RhO}_{3}$ up to $P_{c 1}$ is compatible with hydrostatic pressure conditions, whereas at higher pressures the system progressively moves toward the behavior expected under uniaxial pressure. The uniaxial pressure accounts for the formation of zigzag chains instead of dimers, although it does not fully account for the evolution of the longer $\mathrm{Rh}-\mathrm{Rh}$ bonds that evolve smoothly in the simulation but show a steplike anomaly experimentally (Fig. 6).

At ambient conditions, $\mathrm{Li}_{2} \mathrm{RhO}_{3}$ is an insulator, as shown in Ref. [14]. Our calculated density of states (DOS) for the experimentally obtained structures at $25.2 \mathrm{GPa}$ (Fig. 12) show that unlike other dimerized phases in $\alpha-\mathrm{Li}_{2} \mathrm{IrO}_{3}$ and $\alpha-\mathrm{RuCl}_{3}$, in $\mathrm{Li}_{2} \mathrm{RhO}_{3}$ the degeneracy between $y z$ and $x z$ orbitals of $\mathrm{Rh} d$ states does not get lifted as the symmetry remains the same. Moreover, the system probably becomes metallic under pressure due to the formation of zigzag chains, which provide new hopping pathways. further investigation. Experimental pressure conditions in a DAC with helium as the pressure-transmitting medium are

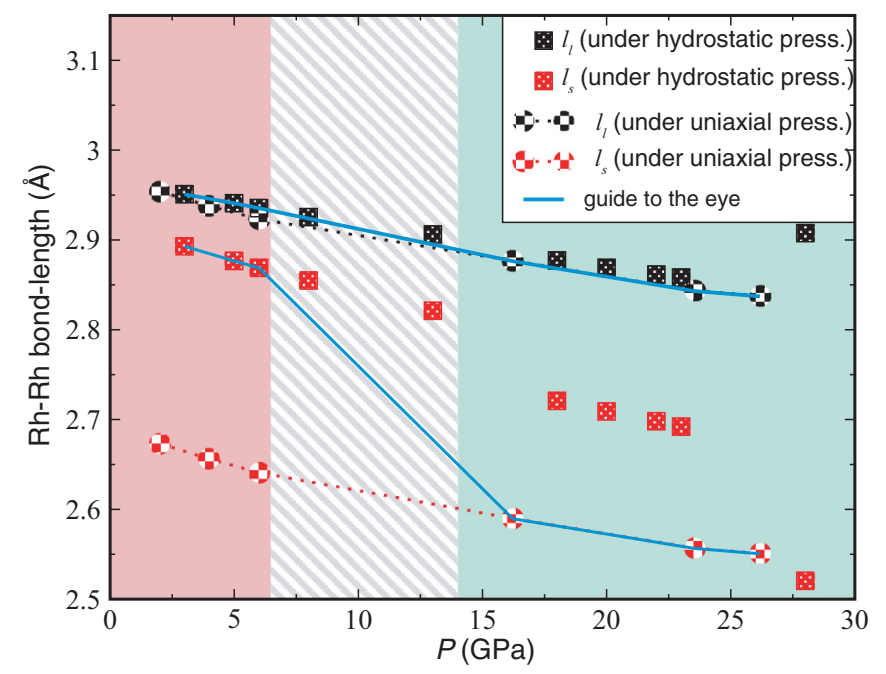

FIG. 11. The evolution of $\mathrm{Rh}-\mathrm{Rh}$ bond lengths (calculated within $\mathrm{GGA}+\mathrm{SOC}+U)$ as a function of pressure. Red and green shaded regions represent hydrostatic and uniaxial pressure regimes, respectively. For comparison with the experimental data, the blue line is drawn as a guide to follow the transition from hydrostatic to uniaxial pressure. 


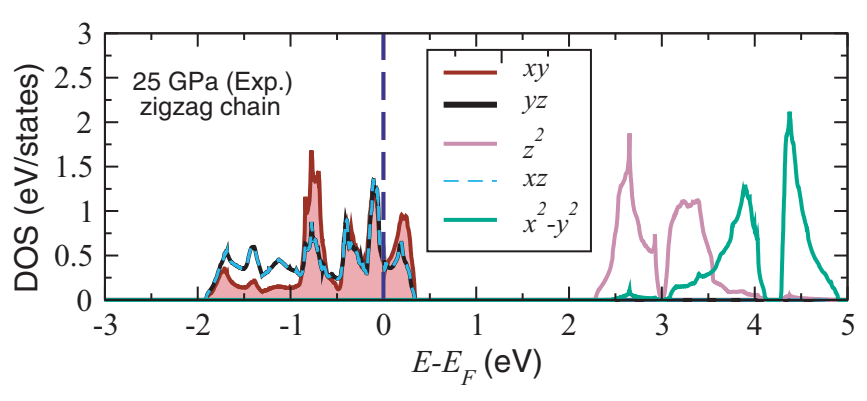

FIG. 12. Orbital-projected DOS for the Rh $d$ orbitals in the experimental structure with $C 2 / m$ symmetry at $25.5 \mathrm{GPa}$, calculated within the GGA $+\mathrm{SOC}+U$ scheme with $U=1.5 \mathrm{eV}$.

expected to be hydrostatic [47]. Therefore, we consider the nature of the $\mathrm{Li}_{2} \mathrm{RhO}_{3}$ sample as a more plausible reason. In particular, stacking faults that occur, on average, every six to seven layers could act as a local strain and affect the evolution of the structure under pressure. Our data show that the concentration of stacking faults in $\mathrm{Li}_{2} \mathrm{RhO}_{3}$ is higher than in the polycrystalline samples of $\alpha-\mathrm{Li}_{2} \mathrm{IrO}_{3}$ and in single crystals of $\alpha-\mathrm{Li}_{2} \mathrm{IrO}_{3}$ that were used in our previous study [15]. Interestingly, $\mathrm{Na}_{2} \mathrm{IrO}_{3}$ shows different pressure evolutions of the crystal structure in powders [48] and single crystals [49]. Given the proclivity of $\mathrm{Na}_{2} \mathrm{IrO}_{3}$ for the formation of stacking faults, a similar mechanism may be operative there and deserves further systematic investigation.

\section{CONCLUSION}

In contrast to $\alpha-\mathrm{Li}_{2} \mathrm{IrO}_{3}$ and $\alpha-\mathrm{RuCl}_{3}$, where a dimerized triclinic phase is stabilized under pressure, $\mathrm{Li}_{2} \mathrm{RhO}_{3}$ retains its ambient-pressure monoclinic $C 2 / m$ symmetry and develops zigzag chains of short $\mathrm{Rh}-\mathrm{Rh}$ bonds. This structural phase transition is not abrupt since traces of the low-pressure phase can still be found even at the highest pressure of $25.2 \mathrm{GPa}$, but above $\approx 14 \mathrm{GPa}$ the high-pressure phase is dominant. Our density-functional calculations suggest that such a behavior is not anticipated in $\mathrm{Li}_{2} \mathrm{RhO}_{3}$ under hydrostatic pressure, where conventional dimerization should occur. On the other hand, uniaxial pressure may explain the experimental observations and promote the formation of zigzag chains instead of dimers.

\section{ACKNOWLEDGMENTS}

We thank the ESRF, Grenoble, for the provision of beam time at ID15B. D.K., R.V., A.A.T., and P.G. acknowledge financial support from the Deutsche Forschungsgemeinschaft (DFG), Germany, through TRR 80 (Project No. 107745057), SPP 1666 (Project No. 220179758), TRR 49 (Project No. 31867626), and SFB 1238 (Project No. 277146847). A.J. acknowledges support from the DFG through Grant No. JE 748/1. S.B. thanks S. Winter for helpful discussions. A.A.T. acknowledges financial support from the Federal Ministry for Education and Research via the Sofja-Kovalevskaya Award of the Alexander von Humboldt Foundation, Germany.
[1] A. Kitaev, Anyons in an exactly solved model and beyond, Ann. Phys. (NY) 321, 2 (2006).

[2] G. Jackeli and G. Khaliullin, Mott Insulators in the Strong SpinOrbit Coupling Limit: From Heisenberg to a Quantum Compass and Kitaev Models, Phys. Rev. Lett. 102, 017205 (2009).

[3] J. Chaloupka, G. Jackeli, and G. Khaliullin, Kitaev-Heisenberg Model on a Honeycomb Lattice: Possible Exotic Phases in Iridium Oxides $\mathrm{A}_{2} \mathrm{IrO}_{3}$, Phys. Rev. Lett. 105, 027204 (2010).

[4] S. K. Choi, R. Coldea, A. N. Kolmogorov, T. Lancaster, I. I. Mazin, S. J. Blundell, P. G. Radaelli, Y. Singh, P. Gegenwart, K. R. Choi, S.-W. Cheong, P. J. Baker, C. Stock, and J. Taylor, Spin Waves and Revised Crystal Structure of Honeycomb Iridate $\mathrm{Na}_{2} \mathrm{IrO}_{3}$, Phys. Rev. Lett. 108, 127204 (2012).

[5] K. W. Plumb, J. P. Clancy, L. J. Sandilands, V. V. Shankar, Y. F. $\mathrm{Hu}, \mathrm{K} . \mathrm{S}$. Burch, H.-Y. Kee, and Y.-J. Kim, $\alpha-\mathrm{RuCl}_{3}$ : A spinorbit assisted Mott insulator on a honeycomb lattice, Phys. Rev. B 90, 041112(R) (2014).

[6] S. H. Chun, J.-W. Kim, J. Kim, H. Zheng, C. C. Stoumpos, C. D. Malliakas, J. F. Mitchell, K. Mehlawat, Y. Singh, Y. Choi, T. Gog, A. Al-Zein, M. M. Sala, M. Krisch, J. Chaloupka, G. Jackeli, G. Khaliullin, and B. J. Kim, Direct evidence for dominant bond-directional interactions in a honeycomb lattice iridate $\mathrm{Na}_{2} \mathrm{IrO}_{3}$, Nat. Phys. 11, 462 (2015).

[7] S. M. Winter, A. A. Tsirlin, M. Daghofer, J. van den Brink, Y. Singh, P. Gegenwart, and R. Valentí, Models and materials for generalized Kitaev magnetism, J. Phys.: Condens. Matter 29, 493002 (2017).
[8] Y. Luo, C. Cao, B. Si, Y. Li, J. Bao, H. Guo, X. Yang, C. Shen, C. Feng, J. Dai, G. Cao, and Z.-an Xu, $\mathrm{Li}_{2} \mathrm{RhO}_{3}$ : A spinglassy relativistic Mott insulator, Phys. Rev. B 87, 161121(R) (2013).

[9] P. Khuntia, S. Manni, F. R. Foronda, T. Lancaster, S. J. Blundell, P. Gegenwart, and M. Baenitz, Local magnetism and spin dynamics of the frustrated honeycomb rhodate $\mathrm{Li}_{2} \mathrm{RhO}_{3}$, Phys. Rev. B 96, 094432 (2017).

[10] J. A. Sears, M. Songvilay, K. W. Plumb, J. P. Clancy, Y. Qiu, Y. Zhao, D. Parshall, and Y.-J. Kim, Magnetic order in $\alpha-\mathrm{RuCl}_{3}$ : A honeycomb-lattice quantum magnet with strong spin-orbit coupling, Phys. Rev. B 91, 144420 (2015).

[11] A. Banerjee, C. A. Bridges, J.-Q. Yan, A. A. Aczel, L. Li, M. B. Stone, G. E. Granroth, M. D. Lumsden, Y. Yiu, J. Knolle, S. Bhattacharjee, D. L. Kovrizhin, R. Moessner, D. A. Tennant, D. G. Mandrus, and S. E. Nagler, Proximate Kitaev quantum spin liquid behavior in a honeycomb magnet, Nat. Mater. 15, 733 (2016).

[12] S. C. Williams, R. D. Johnson, F. Freund, S. Choi, A. Jesche, I. Kimchi, S. Manni, A. Bombardi, P. Manuel, P. Gegenwart, and R. Coldea, Incommensurate counterrotating magnetic order stabilized by Kitaev interactions in the layered honeycomb $\alpha-\mathrm{Li}_{2} \mathrm{IrO}_{3}$, Phys. Rev. B 93, 195158 (2016).

[13] V. M. Katakuri, S. Nishimoto, I. Rousochatzakis, H. Stoll, J. van den Brink, and L. Hozoi, Strong magnetic frustration and anti-site disorder causing spin-glass behavior in honeycomb $\mathrm{Li}_{2} \mathrm{RhO}_{3}$, Sci. Rep. 5, 14718 (2015). 
[14] I. I. Mazin, S. Manni, K. Foyevtsova, H. O. Jeschke, P. Gegenwart, and R. Valentí, Origin of the insulating state in honeycomb iridates and rhodates, Phys. Rev. B 88, 035115 (2013).

[15] V. Hermann, M. Altmeyer, J. Ebad-Allah, F. Freund, A. Jesche, A. A. Tsirlin, M. Hanfland, P. Gegenwart, I. I. Mazin, D. I. Khomskii, R. Valentí, and C. A. Kuntscher, Competition between spin-orbit coupling, magnetism, and dimerization in the honeycomb iridates: $\alpha-\mathrm{Li}_{2} \mathrm{IrO}_{3}$ under pressure, Phys. Rev. B 97, 020104(R) (2018).

[16] J. P. Clancy, H. Gretarsson, J. A. Sears, Y. Singh, S. Desgreniers, K. Mehlawat, S. Layek, G. Kh. Rozenberg, Y. Ding, M. H. Upton, D. Casa, N. Chen, J. Im, Y. Lee, R. Yadav, L. Hozoi, D. Efremov, J. van den Brink, and Y.-J. Kim, Pressure-driven collapse of the relativistic electronic ground state in a honeycomb iridate, npj Quantum Mater. 3, 35 (2018)

[17] G. Bastien, G. Garbarino, R. Yadav, F. J. Martinez-Casado, R. Beltrán Rodríguez, Q. Stahl, M. Kusch, S. P. Limandri, R. Ray, P. Lampen-Kelley, D. G. Mandrus, S. E. Nagler, M. Roslova, A. Isaeva, T. Doert, L. Hozoi, A. U. B. Wolter, B. Büchner, J. Geck, and J. van den Brink, Pressure-induced dimerization and valence bond crystal formation in the Kitaev-Heisenberg magnet $\alpha-\mathrm{RuCl}_{3}$, Phys. Rev. B 97, 241108(R) (2018).

[18] T. Biesner, S. Biswas, W. Li, Y. Saito, A. Pustogow, M. Altmeyer, A. U. B. Wolter, B. Büchner, M. Roslova, T. Doert, S. M. Winter, R. Valentí, and M. Dressel, Detuning the honeycomb of $\alpha-\mathrm{RuCl}_{3}$ : Pressure-dependent optical studies reveal broken symmetry, Phys. Rev. B 97, 220401(R) (2018)

[19] S. Manni, Synthesis and investigation of frustrated Honeycomb lattice iridates and rhodates, Ph.D. thesis, University of Goettingen, 2014.

[20] V. Petříček, M. Dušek, and L. Palatinus, Crystallographic Computing System JANA2006: General features, Z. Kristallogr. Cryst. Mater. 229, 345 (2014).

[21] R. A. Young, The Rietveld Method, reprint, International Union of Crystallography Monographs on Crystallography Vol. 5 (Oxford University Press, Oxford, 2002).

[22] R. B. Von Dreele, M. R. Suchomel, and B. H. Toby, Compute X-ray Absorption, Argonne National Laboratory (2013), https://11bm.xray.aps.anl.gov/absorb/absorb.php.

[23] P. E. Blöchl, Projector augmented-wave method, Phys. Rev. B 50, 17953 (1994).

[24] G. Kresse and J. Hafner, Ab initio molecular dynamics for liquid metals, Phys. Rev. B 47, 558 (1993).

[25] S. L. Dudarev, G. A. Botton, S. Y. Savrasov, C. J. Humphreys, and A. P. Sutton, Electron-energy-loss spectra and the structural stability of nickel oxide: An LSDA $+U$ study, Phys. Rev. B 57, 1505 (1998).

[26] K. Koepernik and H. Eschrig, Full-potential nonorthogonal local-orbital minimum-basis band-structure scheme, Phys. Rev. B 59, 1743 (1999).

[27] M. J. O'Malley, H. Verweij, and P. M. Woodward, Structure and properties of ordered $\mathrm{Li}_{2} \mathrm{IrO}_{3}$ and $\mathrm{Li}_{2} \mathrm{PtO}_{3}$, J. Solid State Chem. 181, 1803 (2008).

[28] F. Freund, S. C. Williams, R. D. Johnson, R. Coldea, P. Gegenwart, and A. Jesche, Single crystal growth from separated educts and its application to lithium transition-metal oxides, Sci. Rep. 6, 35362 (2016).
[29] J. Bréger, M. Jiang, N. Dupré, Y. S. Meng, Y. Shao-Horn, G. Ceder, and C. P. Grey, High-resolution X-ray diffraction, DIFFaX, NMR and first principles study of disorder in the $\mathrm{Li}_{2} \mathrm{MnO}_{3}-\mathrm{Li}\left[\mathrm{Ni}_{1 / 2} \mathrm{Mn}_{1 / 2}\right] \mathrm{O}_{2}$ solid solution, J. Solid State Chem. 178, 2575 (2005).

[30] M. Casas-Cabanas, J. Rodríguez-Carvajal, J. Canales-Vázquez, Y. Laligant, P. Lacorre, and M. R. Palacín, Microstructural characterisation of battery materials using powder diffraction data: DIFFaX, FAULTS and SH-FullProf approaches, J. Power Sources 174, 414 (2007).

[31] F. D. Murnaghan, The Compressibility of Media under Extreme Pressures, Proc. Natl. Acad. Sci. USA 30, 244 (1944).

[32] V. Todorova and M. Jansen, Synthesis, Structural Characterization and Physical Properties of a New Member of Ternary Lithium Layered Compounds $-\mathrm{Li}_{2} \mathrm{RhO}_{3}$, Z. Anorg. Allg. Chem. 637, 37 (2011).

[33] F. Ye, S. Chi, H. Cao, B. C. Chakoumakos, J. A. FernandezBaca, R. Custelcean, T. F. Qi, O. B. Korneta, and G. Cao, Direct evidence of a zigzag spin-chain structure in the honeycomb lattice: A neutron and $\mathrm{x}$-ray diffraction investigation of singlecrystal $\mathrm{Na}_{2} \mathrm{IrO}_{3}$, Phys. Rev. B 85, 180403(R) (2012).

[34] E. S. Bale, The Structure of Rhodium, Platinum Metals Rev. 2, 61 (1958).

[35] K. Mehlawat and Y. Singh, First-order magnetostructural transition in single crystals of the honeycomb lattice ruthenate $\mathrm{Li}_{2} \mathrm{RuO}_{3}$, Phys. Rev. B 95, 075105 (2017).

[36] S. A. J. Kimber, I. I. Mazin, J. Shen, H. O. Jeschke, S. V. Streltsov, D. N. Argyriou, R. Valentí, and D. I. Khomskii, Valence bond liquid phase in the honeycomb lattice material $\mathrm{Li}_{2} \mathrm{RuO}_{3}$, Phys. Rev. B 89, 081408(R) (2014).

[37] S. M. Winter, Y. Li, H. O. Jeschke, and R. Valentí, Challenges in design of Kitaev materials: Magnetic interactions from competing energy scales, Phys. Rev. B 93, 214431 (2016)

[38] A. Ertl, J. M. Hughes, F. Pertlik, F. F. Foit, S. E. Wright, F. Brandstatter, and B. Marler, Polyhedron distortions in tourmaline, Can. Mineral. 40, 153 (2002).

[39] E. S. Kim, S. H. Kim, and K. H. Yoon, Dependence of Thermal Stability on Octahedral Distortion of $(1-x)$ $\left(\mathrm{Ca}_{0.3} \mathrm{Li}_{0.119} \mathrm{Sm}_{0.427}\right) \mathrm{TiO}_{3}-x \mathrm{LnAlO}_{3}(\mathrm{Ln}=\mathrm{Nd}, \mathrm{Sm})$ Ceramics, J. Ceram. Soc. Jpn. Supplement 112, S1645 (2004).

[40] T. Hogan, X. Wang, H. Chu, D. Hsieh, and S. D. Wilson, Doping-driven structural distortion in the bilayer iridate $\left(\mathrm{Sr}_{1-x} \mathrm{La}_{x}\right)_{3} \mathrm{Ir}_{2} \mathrm{O}_{7}$, Phys. Rev. B 95, 174109 (2017).

[41] J. C. Wang, J. Terzic, T. F. Qi, F. Ye, S. J. Yuan, S. Aswartham, S. V. Streltsov, D. I. Khomskii, R. K. Kaul, and G. Cao, Latticetuned magnetism of $\mathrm{Ru}^{4+}\left(4 d^{4}\right)$ ions in single crystals of the layered honeycomb ruthenates $\mathrm{Li}_{2} \mathrm{RuO}_{3}$ and $\mathrm{Na}_{2} \mathrm{RuO}_{3}$, Phys. Rev. B 90, 161110(R) (2014).

[42] H. Kobayashi, R. Kanno, Y. Kawamoto, M. Tabuchi, O. Nakamura, and M. Takano, Structure and lithium deintercalation of $\mathrm{Li}_{2-x} \mathrm{RuO}_{3}$, Solid State Ionics 82, 25 (1995).

[43] G. A. Gehring and K. A. Gehring, Co-operative Jahn-Teller effects, Rep. Prog. Phys. 38, 1 (1975).

[44] M. D. Kaplan and B. G. Vekhter, Cooperative Phenomena in Jahn-Teller Crystals, Modern Inorganic Chemistry (Springer, Boston, 1995).

[45] Q. Huang, J. L. Soubeyroux, O. Chmaissem, I. N. Sora, A. Santoro, R. J. Cava, J. J. Krajewski, and W. F. Peck, Neutron 
Powder Diffraction Study of the Crystal Structures of $\mathrm{Sr}_{2} \mathrm{RuO}_{4}$ and $\mathrm{Sr}_{2} \mathrm{IrO}_{4}$ at Room Temperature and at $10 \mathrm{~K}$, J. Solid State Chem. 112, 355 (1994).

[46] T. Vogt and D. J. Buttrey, Temperature Dependent Structural Behavior of $\mathrm{Sr}_{2} \mathrm{RhO}_{4}$, J. Solid State Chem. 123, 186 (1996).

[47] S. Klotz, J.-C. Chervin, P. Munsch, and G. Le Marchand, Hydrostatic limits of 11 pressure transmitting media, J. Phys. D 42, 085413 (2009).
[48] X. Xi, X. Bo, X. S. Xu, P. P. Kong, Z. Liu, X. G. Hong, C. Q. Jin, G. Cao, X. Wan, and G. L. Carr, Honeycomb lattice $\mathrm{Na}_{2} \mathrm{IrO}_{3}$ at high pressures: A robust spin-orbit Mott insulator, Phys. Rev. B 98, 125117 (2018).

[49] V. Hermann, J. Ebad-Allah, F. Freund, I. M. Pietsch, A. Jesche, A. A. Tsirlin, J. Deisenhofer, M. Hanfland, P. Gegenwart, and C. A. Kuntscher, High-pressure versus isoelectronic doping effect on the honeycomb iridate $\mathrm{Na}_{2} \mathrm{IrO}_{3}$, Phys. Rev. B 96, 195137 (2017). 